\title{
TOWARDS THE AUTOMATIC 3D PARAMETRIZATION OF NON-PLANAR SURFACES FROM POINT CLOUDS IN HBIM APPLICATIONS
}

\author{
J. Román ${ }^{1 *}$, P. M. Lerones ${ }^{1}$, J. Llamas ${ }^{1}$, E. Zalama ${ }^{2}$, J. Gómez-García-Bermejo ${ }^{2}$ \\ ${ }^{1}$ CARTIF, Parque Tecnológico de Boecillo, Valladolid, Spain - (javrom, pedler, joslla)@ cartif.es \\ ${ }^{2}$ ITAP-DISA, University of Valladolid, Valladolid, Spain- (ezalama, jaigom)@eis.uva.es
}

Commission II, WG II/8

KEY WORDS: HBIM, Façade Parsing, Point Cloud, Cloud to BIM, Cultural Heritage, feature detection, non-planar surfaces

\begin{abstract}
:
3D laser scanning and photogrammetric 3D reconstruction generate point clouds from which the geometry of BIM models can be created. However, a few methods do this automatically for concrete architectural elements, but in no case for the entirety of heritage assets. A novel procedure for the automatic recognition and parametrization of non-planar surfaces of heritage immovable assets is presented using point clouds as raw input data. The methodology is able to detect the most relevant architectural features in a point cloud and their interdependences through the analysis of the intersections of related elements. The non-planar surfaces detected, mainly cylinders, are studied in relation to the neighbouring planar surfaces present in the cloud so that the boundaries of both the planar and the non-planar surfaces are accurately defined. The procedure is applied to the emblematic Castle of Torrelobatón, located in Valladolid (Spain) to allow the cataloguing of required elements, as illustrative example of the European defensive architecture from the Middle age to the Renaissance period. Results and conclusions are reported to evaluate the performance of this approach.
\end{abstract}

\section{INTRODUCTION}

For the last decade both laser and photogrammetric 3D reconstruction have proven to be suitable technologies for the digitization of immovable heritage assets. They still are relevant documentation techniques as they are involved in a dailygrowing number of restoration and conservation projects. What is more, recent technological advances in the fields of optics, signal processing, electronics and computers have improved the capabilities of these technologies allowing professionals of the AEC industry and researchers to obtain dense point clouds and triangle meshes with higher accuracy and level of detail. According to Barrile (2015), "the world of cultural heritage is experiencing a phase of promotion and development of its assets thanks to the progress of survey techniques".

It should be pointed out that using point clouds as a reference to create the parametrical geometry required by the Building Information Models (BIMs) is time-consuming, costly and error-prone while the accuracy of the reconstructed models needs to be guaranteed (Xiong, 2013; Chen, 2014; Stambler, 2014; Macher, 2017; Yang, 2018). Moreover, the result produced by trained modellers may differ significantly from each other (Xiong, 2013). On the other hand, the European Directive 2014/24/EU imposes BIM Level 2 for government centrally procured projects. Heritage is not ruled out and BIM is becoming adopted in a growing-pace for restoration planning, facility management and documentation purposes since BIM models allows to collect, organize and manage all the assets' data into a single intelligent carrier of information (Historic
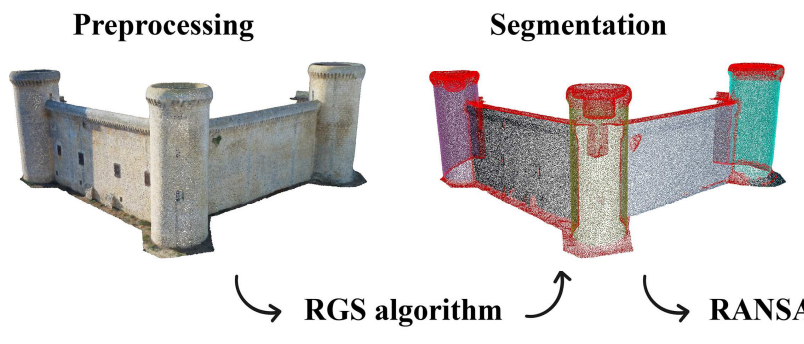

$\longrightarrow$ RANSAC algorithm

England, 2017; López, 2017). The model is not just a virtual representation of the construction, but a collection of "smart objects" with parametric intelligence (Pocobelli, 2018). Thus, 3D modelling of as-built heritage immovable assets from point clouds, widely known as Scan-to-BIM, is a key issue that has to be addressed during the development of heritage projects since the BIM follow a bottom-up process: parametric modelling of the specific parts for the studied heritage asset, and then integrating the additional information into the model.

The automatic reconstruction of $3 \mathrm{D}$ models has been a challenging research topic and from the early beginnings numerous research papers based on different reconstruction methods have been published (Grün, 1995; Peternell, 2003; Pu, 2007; Pu, 2009; As Habib, 2010; Haala, 2010; Xiong, 2013; Cheng, 2014; Zolanvari, 2016; Yang, 2018). Even though a wide variety of modelling techniques ranging from nearly manual to almost automatic have been developed (Ochmann, 2016; López, 2018), automatic reconstruction still remains a major topic in which experts are putting their efforts on.

Many researchers claim that the complete automation of the cloud-to-BIM methodology is not yet currently viable, and expert intervention is required since artificial intelligence has not been yet developed to a point in which it can make architectural assumptions on its own (Tommasi, 2016; Arayici, 2017; Bruno, 2018). The most advanced reconstruction algorithms make assumptions about the shape and the main features of the analysed building. It is not an overstatement to say that there is no complete, end-to-end automated process for

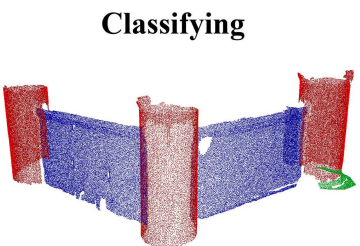

Parametrization



Projection-based
intersection algorithm

Figure 1: Overview of the proposed method. From left to right. Preprocessing; Segmentation; Classifying; Parametrization 
generating the parametric models.

In one hand, there is prior work on modelling the exteriors of buildings, but mostly has been focused on the reconstruction of planar façades with, at best, openings like windows and doors. Authors like Wan (2012), Wang (2015) and Li (2017) proposed algorithms to model building exteriors recognizing walls as planes. These resulting planes are grouped together into a single building volume. Despite being able to accurately detect the main surfaces, building models are overly-simplified and in any case, the representation is reduced to planar features. TruongHong (2012) and Zolanvari (2018) proposed single and multifaçade slicing methods. After being provided by a principal plane, the techniques slice the façade into limited portions. Though they are able to detect the overall boundary of masonry brick walls and each opening area's boundaries (windows and doors), their algorithms presumes the existence of regular openings in almost planar walls. Finally, Xiong (2013), Stambler (2014), Ochman (2015) and Jung (2018) use both interior and exterior point clouds of the building to generate the more likely floor plant distribution by means of a statistical hypothesis about the placement of the walls and its connectivity. Despite accurately detecting the distribution of the rooms, the hypothesis used can hardly be applied to immovable heritage assets such as the uniformity of the walls, which must be planes to properly define the divisions.

In the other hand, there are commercial plug-ins for efficiently using as-built point cloud data captured of existing buildings by laser scanners directly within BIM environments. These plugins are conceived to let users get the advantages of a highperformance point cloud application directly within these applications, but restricted to specific point cloud formats according to the brand of the laser scanner. In any case, such a kind of plug-ins are only oriented to 3D laser scanning data on recent building and civil works, but not to the built heritage, so they do not expressly meet related needs.

The extensive research that has been done on this topic leads to the conclusion that no single previous proposal was able to automatically parametrize non-planar surfaces which were part of heritage buildings. To overcome the limitations of these approaches, it is presented a novel method upon the state of the art to automatically convert raw $3 \mathrm{D}$ point cloud data with both planar and non-planar surfaces into a 3D model. The method automatically reconstructs planar walls as well as round towers, modelled as planes and cylinders respectively, while simultaneously taking into account local connectivity of all elements. These elements, which are present in a wide range of heritage buildings, can be used for modelling most part of this type of architectural complexes. However, it must be noticed that the vast majority of the architectural geometries making the built heritage can be also modelled by means of geometrical primitives such as spheres, cones or torus which can be easily integrated into the proposed methodology upon request. The reconstruction is formulated as a segmentation problem which in successive steps classifies and parametrizes the detected architectural elements using a statistical model fitting. Once the segments have been properly classified a further parametrization step is carried out, so both the boundaries of the classified features and the common edges between planes and cylinders can be determined. Finally, it is worth mentioning that parametrized features are intended to be a basic workspace where more complex and detailed objects can be incorporated in a later stage of the digital reconstruction process.
The proposed algorithm has been applied to a real case study: the Castle of Torrelobatón in Valladolid, Spain, as representative example of the defensive architecture from the Middle age to the Renaissance in Europe (López, 2018). The point cloud (XYZ and RGB coordinates) was collected using photogrammetric $3 \mathrm{D}$ reconstruction and exported in a later step to a triangular mesh. Several datasets containing representative architectonic features are extracted from the cloud and analysed so that accuracy and overall performance of the developed algorithm can be evaluated.

\section{METHODOLOGY}

The approach comprises four main steps (see Figure 1). First, the raw point cloud is pre-processed by removing noisy data and downsizing the number of points. Once the pre-processing is completed, a region growing segmentation algorithm is applied to divide the downsized cloud into its constituent parts so they can be processed independently. Then, a model fitting classifying technique determines whether the segments can be interpolated by planes or cylinders. Finally, the segments are parametrized and the local connectivity of the detected features is established so the building geometry can be successfully extracted. Whereas pre-processing and segmentation are widely used algorithms in point cloud treating applications, the classifying and dependency analysis steps are a noteworthy advance upon the latest automatic modelling developments. The aforementioned steps are detailed in the following paragraphs.

\subsection{Data pre-processing}

2.1.1 Filtering: The point cloud data, once loaded, is filtered by a statistical algorithm minimizing potential problems to avoid an inaccurate final result or even a failure in following processing steps. In most cases, statistical filtering algorithms compare the value of a property of one of the points with the mean value of the property within the point cloud removing those which fall outside a threshold range. It is been used a distance-based criteria to remove distant points from their neighbouring points. First, the algorithm determines the mean distance of each point to its neighbours. Then, an overall mean distance for the whole point cloud is calculated. Assuming a Gaussian distribution, all the points falling outside of the confidence interval defined by the global mean distance and standard deviation are considered noisy and, therefore, removed from the point cloud.

2.1.2 Resampling: The resampling algorithm modifies the number of points of the input point cloud without changing the geometrical dimension or the resolution of the initial data set by adding or removing points from the digitized scenes. Resampling is used to decrease the point cloud density in order to boost the processing speed and ensuing computationally intensive steps. The algorithm generates an octal tree from the data set, a uniform recursive voxel grid in which $3 \mathrm{D}$ space is divided. Then, all the points belonging to the same voxel of the grid are replaced by its centroid. Despite not being necessary, this step greatly diminishes the overall computation time when compression parameters are properly adjusted.

\subsection{Segmentation}

The local connectivity between primitives is a key topological problem which must be addressed for the correct interpretation of the cloud. Analysing the whole point cloud at one time is an almost impossible task which may lead to very long processing times, highly inaccurate results or, failures in most cases. The 
segmentation of a point cloud consists on classifying every single point of the data set into multiple regions so points belonging to the same region will share the same properties.

Surface normals, an important property of geometric surfaces, have to be estimated to correctly segment the point cloud. The direction of the surface normal at a certain point of a given mathematical surface can be retrieved as the vector perpendicular to the surface in the analysed point. As point clouds contain discrete superficial samples of the real scene, determining the normal to a point has been approximated by the problem of estimating the normal of the plane tangent to the surface in that point, which in expressed in mathematical terms as a least-square plane fitting estimation problem. Once the surface normals are calculated, a region growing segmentation algorithm is applied to merge points close enough in terms of surface normals orientation into a point cloud. Hence, the data set is divided into constituent parts, being each region a collection of points considered as part of the same surface.

The applied algorithms are pretty straight-forward so far and parameter tuning is not required with the exception of the resampling algorithm, which can be parametrized by the leaf size of the generated octree. However, segmentation phase requires a little few setting parameters: a threshold value for the deviation of neighbouring normals, a threshold value for the curvature and optionally, a cut-off value for the lower number of points that a region must have. The optimal values were explored in an experimental iterative process and for an optimal computation performance, the minimum size of the segment was related with a percentage of the total number of points of the cloud.

\subsection{Classifying}

The aim of the classifying stage is to assign a label to every single segmented point cloud extracted from the initial dataset based on its outer shape. It is noteworthy that, due to the sheer complexity of the problem, the algorithm makes a little few assumptions about the shape of the point clouds analysed. Hence, the algorithm is focused on the analysis of walls and towers even though more advanced architectonic features could be further added such as domes or vaults as discussed in conclusions section. The different segments are labelled according to the mathematical model that best fits the points of the region, namely: 1) planes; 2) cylinders; 3) and undefined. The classifying step relies on the statistical algorithm RANSAC (RANdom SAmple Consensus; Fischler, 1981), which is capable of estimating the parameters of a fitted model through an iterative process, assuming that the starting data is comprised of both inliers and outliers. Inliers (points closer to the model than a certain threshold distance specified by the user) are used to estimate the model, while outliers are put aside during the estimation process.

The advantage of RANSAC relies on the ability to do a robust estimation of the model parameters with empty areas or a relevant amount of noise in the initial dataset. However, the upper bound on the number of iterations must be manually set, and the final outcome may not be optimal or not even fit the points in a good way if the chosen model was inadequate. Further, a high percentage of points adjusted can be a misleading metric since it does not necessarily mean that the selected model is the best fitted to the dataset. To avoid this major problem a classifying algorithm has been implemented that selects the best fitting model between the aforementioned. The parameters of both the fitting plane and cylinder are estimated for every single dataset and the model that explains the largest amount of points is selected if a high enough percentage of them are considered inliers, a parameter manually specified by the user. Otherwise, the segment is classified as undefined. Just the model is validated, the inliers are projected onto the selected surface and saved for later usage while the outliers are discarded.

\subsection{Parametrization}

While planes are parametrized by its scalar equation, cylinders are represented by the point-slope equation of its axis and the radius. However, these equations define infinite geometrical surfaces and limits need to be established.

Two assumptions are made about the walls: 1) they can be modelled as planes; 2) they have rectangular shape. This simplification eases and robusts the connectivity-establishing process and, a coarse simplification is made, without loss of information since the proposed approach tries to recreate the "as-build" 3D model as opposed to the "as-is" model. To parameterize the walls, a multi-step approach is applied with the aim of determining the four vertices of the rectangle. First, the boundaries of the clouds related to each wall are enlarged. During the segmentation of the cloud the bordering points of the segments were excluded due to the irregularities of the surface normals, reducing the overall area of the cloud compared to the surface of the theoretical wall.

The cloud is enlarged by projecting points onto the edges defined by the intersections of the current analysed plane with the rest of detected walls. Then, points from both the cloud related to the plane and the points of the rest of the walls are projected onto the shared edge. In order to optimize the computational performance, only the bordering points of the walls are taken into account during the calculations. If the two projected datasets are close enough to the points of the wall, the projected points are added to the main cloud. Enlarging walls close to towers is done in a similar projection method approach. So, the bordering points of the walls are projected onto the cylinders and this auxiliary data set is projected a second time but onto the plane to ensure its coplanarity with the wall. If the projected points are close enough to the wall, the auxiliary data set is added to the main cloud.

Next, the vertices of the wall are determined using underlying information contained in the enlarged cloud. The algorithm iterates through the points and keeps the maximum and minimum registered values of the XYZ coordinates. Then, four auxiliary vertices are generated as candidates for the two lower vertices of the plane combining the values of the registered coordinates $\mathrm{XY}$ always keeping constant the value of the coordinate $\mathrm{Z}$. The Euclidean distance between the vertices and the plane is determined and the two farthest are discarded. The upper vertices are obtained replacing the value of the $\mathrm{Z}$ coordinate of the two remaining vertices for the maximum $\mathrm{Z}$ value registered. Finally, the four vertices are projected onto the plane to ensure its coplanarity and saved for further refinement using the mathematical equations of the edges. By intersecting them it is possible to replace previously calculated vertices with the intersections, which have a higher accuracy. The new points are compared with the original vertices and if one of them is close enough to one intersection, a replacement takes place (in which case the coordinates of the other vertices are modified to keep the proportions of the rectangle). The flowchart of the proposed methodology is shown in Figure 2. 


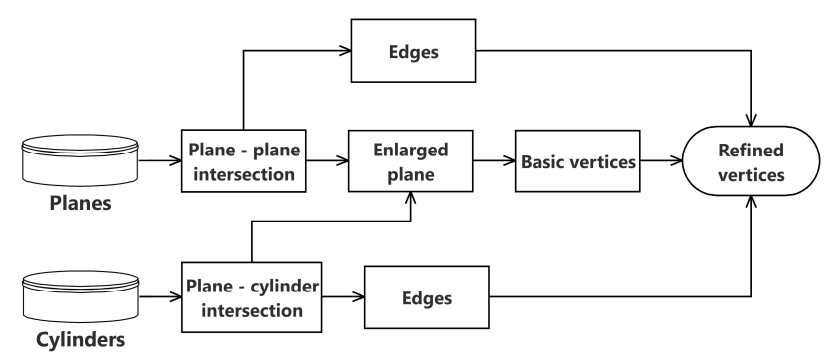

Figure 2: Flowchart of the proposed methodology to calculate the vertices of the walls.

To parametrize the towers, it is assumed the premise that the towers can be modelled as cylinders. The RANSAC parametrizes this geometrical feature with the point-slope of the axis and the length of the radius. If the slope deviates more than a certain angle with respect to the $\mathrm{OZ}$ axis, the cylinder is discarded as candidate to tower. In order to establish the limits of the cylinder, the centre of their bases are calculated. First the points with maximum and minimum value of the $\mathrm{Z}$ coordinate are determined by iterating through the points of the cloud and two planes perpendicular to the OZ axis are defined containing one of each points respectively. Then, the coordinates of the centres of the bases are obtained intersecting the axis of the cylinder with these planes. So, having the radius, the two centres of the bases and the slope, the limits of the cylinder can be well-stablished. It must be noticed that the cylinders are defined on their own, as opposed to the planes that are defined according to the dimensions of their neighbouring planes and cylinders.

Finally, both planes and cylinders are saved as IFC2x3 wall type models so they can be imported in BIM modelling environments. Since the geometry of the detected features was generated following the requirements of this standard no further conversions are required when importing the models.

\subsection{Results}

The results obtained when applying the described algorithms to a real-world point cloud of the Castle of Torrelobatón as well as a synthetic cloud are described in the next paragraph. The two point clouds were selected to stress the methodology by testing case-corner situations such as wide gaps between the detected

\begin{tabular}{|c|c|c|c|c|c|c|c|}
\hline \multirow[t]{2}{*}{${ }^{\circ}$ Step } & \multirow[t]{2}{*}{ Sub-step } & \multirow[t]{2}{*}{ Parameter(s) } & \multicolumn{2}{|c|}{ Real-world } & \multicolumn{2}{|l|}{ Synthetic } & \multirow[t]{2}{*}{ Remark } \\
\hline & & & Time (s) & Value & Time (s) & Value & \\
\hline Loading & & - & 1.45 & - & 8.03 & - & - \\
\hline \multirow[t]{2}{*}{ Pre-processing } & Filtering & Neighbouring points & 0.08 & 50 & 0.75 & 50 & Assumed \\
\hline & Resizing & Leaf size & 0.04 & 0.60 & 0.18 & 0.4 & User-specified \\
\hline \multirow[t]{3}{*}{ Segmentation } & Surface normal & Neighbouring points & 0.58 & 50 & 2.06 & 50 & Assumed \\
\hline & Segmentation & Smoothness threshold & 0.50 & 0.04 & 1.67 & 0.02 & User-specified \\
\hline & & Minimum segment $\%$ & & 1 & & 1 & User-specified \\
\hline \multirow[t]{3}{*}{ Classifying } & & Plane inlier threshold & 0.14 & 0.30 & 0.39 & 0.25 & User-specified \\
\hline & & Cylinder inlier threshold & & 0.30 & & 0.25 & User-specified \\
\hline & & Minimum inliers percentage & & 75 & & 85 & User-specified \\
\hline \multirow[t]{2}{*}{ Parametrization } & & Plane to plane threshold & 0.05 & 2 & 1.25 & 2.5 & User-specified \\
\hline & & Plane to cylinder threshold & & 2 & & 1.5 & User-specified \\
\hline
\end{tabular}

Table 2: Parameters selection and time consumption for each modelling step

features and multi-encounter primitive situations. The trials were conducted on a Windows 10, Intel(R) Core i5 3470@3.2 $\mathrm{GHz}$ CPU with 16GB of memory. The processing time spent on each reconstruction step and the parameter selection is detailed in Table 2 for both the real-world and the synthetic point clouds.

The real-world point cloud has 177.397 points and contains three towers and three walls and has been prepared to prove the performance of the application in a real world environment (see Figure 3a). During the first stage, the pre-processing algorithms decreased the size of the cloud in a nearly forty percent. The segmentation process was able to extract seven segments from the pre-processed cloud and 36.957 points $(36.40 \%)$ were left unsegmented (Figure $3 \mathrm{~b}$ ). For a segment to be considered as part of a category, a $75 \%$ of its points had to be considered as inliers (Figure 3d, outliers in grey colour) as empirical rule of thumb based on the overall adjustment achieved for the RANSAC for both planes and cylinders and also taking into account the upper fitting percentage achieved by the undefined segments. With this settings, the classifying algorithm correctly labelled three of the samples as planes (P), three as cylinders (C) and one of them was classified as undetermined (U) as seen in Table 1 (see also Figure 3c). In this table the percentage of inliers is presented as well as their overall mean distance to the fitted model.

\begin{tabular}{llllllll}
\hline Segment & 1 & 2 & 3 & 4 & 5 & 6 & 7 \\
\hline Plane $\%$ & 81 & 10 & 11 & 83 & 100 & 12 & 59 \\
Cylinder $\%$ & 0 & 91 & 93 & 25 & 46 & 99 & 36 \\
Label & $\mathrm{P}$ & $\mathrm{C}$ & $\mathrm{C}$ & $\mathrm{P}$ & $\mathrm{P}$ & $\mathrm{C}$ & $\mathrm{U}$ \\
$\mu(\mathrm{mm})$ & 42 & 72 & 62 & 48 & 28 & 67 & - \\
$\sigma(\mathrm{mm})$ & 1.6 & 2.2 & 2.5 & 1.6 & 0.8 & 2.0 & - \\
\hline
\end{tabular}

Table 1: Real-world point cloud. Percentage of inliers adjusted for each model, mean distance $(\mu)$ and standard deviation $(\sigma)$.

With regard to the parametrization process, the algorithm was able to accurately detect the encounters of the walls with the cylinders. The local connectivity projection-based method, the relations between the different detected features and the results are represented in Figure $3 \mathrm{f}$.

西


(a)



(c)

(6)

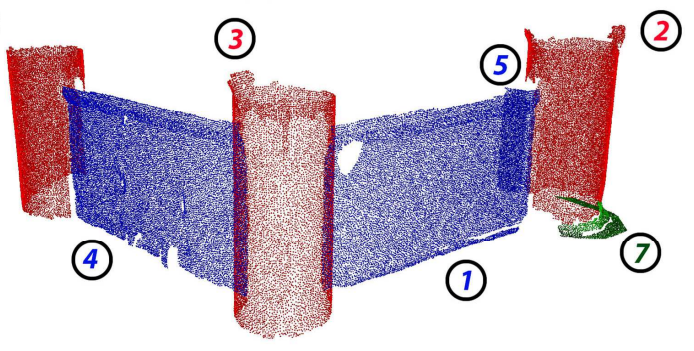

(e)



(b)

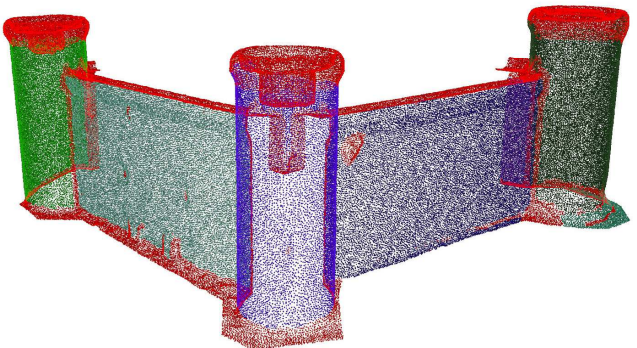

(d)

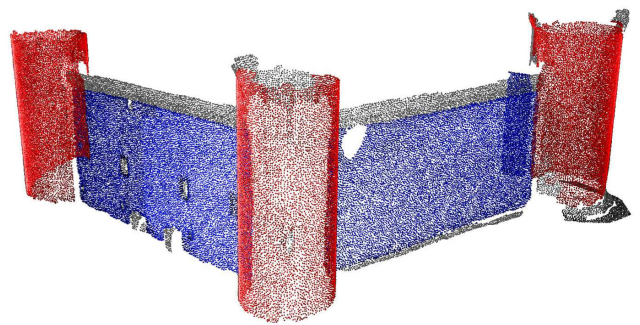

(f)


Figure 3: a) Preprocessed; b) Segmented; c) Primitives (cylinders in red, walls in blue, undefined in green); d) Inliers and outliers (grey); e) Projected inliers; f) Parametrization and improved shape (detail).

Additional architectonic features of the towers may be parameterized exploiting the intrinsic revolution nature of this element. By using information gathered during the parametrization of the cylinders it is possible to characterize the battlements of the tower. The proposed algorithm segments the initial point cloud of the castle applying a filtering algorithm that cuts off points inside a given range along one or more coordinate axis. The points that fall inside a 3D voxel resting on the top centre of the cylinder are filtered, being the side of the cube a parameter related to the radius of the cylinder. Finally, the extracted dataset is projected onto a plane that contains the axis of the cylinder being the bordering points of the dataset the profile of these revolution features. In this manner, the battlements of the towers have been parameterized using the proposed approach. The algorithm was able to extract correctly the profile of the battlements of the towers using the unsegmented points to optimize the computational performance and the final outcome is shown in Figure 4d. (a)

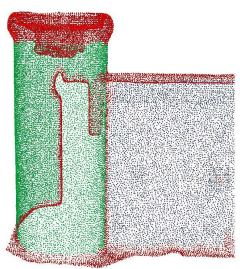

(b)

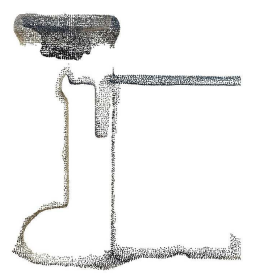

(c)

(d)

Figure 4: a) Segmentation; b) Unsegmented; c) Projection of the extracted revolution points; d) Polygonal profile.

The synthetic point cloud has been populated from representative elements of the castle containing several walls and towers (Figure 5a). The model is relatively simple in terms of the diversity of its categories; it mainly consists of the structural elements under study. One million points were generated for the whole model using subsamples from the original data. The segmentation step generated 31 different datasets and the final parameterized model contains 20 planes and 11 cylinders (see Figure 5b, Figure 5c).

\footnotetext{
* Corresponding author
} 


\begin{tabular}{|c|c|c|c|c|c|c|c|c|c|c|c|c|c|c|c|c|}
\hline Segment & & 0 & 1 & 2 & 3 & 4 & 5 & 6 & 7 & 8 & 9 & 10 & 11 & 12 & 13 & 14 \\
\hline Plane (\%) & & 100 & 100 & 99.7 & 12.6 & 100 & 11.7 & 100 & 100 & 100 & 12.7 & 100 & 99.6 & 46.5 & 13.3 & 100 \\
\hline Cylinder (\%) & & 14.2 & 2.7 & 0,0 & 100 & 0.0 & 100 & 0.0 & 2.4 & 3.0 & 98.3 & 23.4 & 24.3 & 100 & 100 & 13.9 \\
\hline Remark & & & & & & & & & & & & & & $1^{*}$ & & \\
\hline$\mu(\mathrm{mm})$ & & 44.5 & 23.2 & 19.5 & 63.9 & 22.5 & 69.8 & 23.2 & 21.9 & 21.0 & 67.9 & 23.4 & 20.1 & 56.6 & 64.7 & 25.0 \\
\hline$\sigma(\mathrm{mm})$ & & 1.0 & 0.4 & 0.7 & 2.1 & 0.4 & 2.6 & 0.4 & 0.3 & 0.3 & 1.9 & 0.4 & 0.6 & 1.3 & 2.2 & 0.6 \\
\hline Segment & 15 & 16 & 17 & 18 & 19 & 20 & 21 & 22 & 23 & 24 & 25 & 26 & 27 & 28 & 29 & 30 \\
\hline$\%$ Plane & 100 & 100 & 100 & 100 & 30.8 & 22.3 & 19.1 & 71.9 & 38.3 & 100 & 100 & 100 & 99.3 & 99.8 & 100 & 12,4 \\
\hline$\%$ Cylinder & 26.4 & 35.2 & 2.4 & 18.2 & 100 & 100 & 100 & 100 & 99.4 & 11.1 & 12.1 & 17.4 & 22.0 & 18.0 & 19.7 & 100 \\
\hline Remark & & & & & $3^{*}$ & $1^{*}$ & $2^{*}$ & $2^{*}$ & $3^{*}$ & & & & & & & \\
\hline$\mu(\mathrm{mm})$ & 19.9 & 24.4 & 20.2 & 22.9 & 55.3 & 60.5 & 62.6 & 52.4 & 81.0 & 22.7 & 20.9 & 24.2 & 31.5 & 22.0 & 20.3 & 70.9 \\
\hline$\sigma(\mathrm{mm})$ & 0.3 & 0.5 & 0.2 & 0.4 & 1.2 & 1.8 & 1.8 & 0.9 & 3.2 & 0.3 & 0.3 & 0.5 & 1.0 & 0.3 & 0.3 & 3.0 \\
\hline
\end{tabular}

(a)

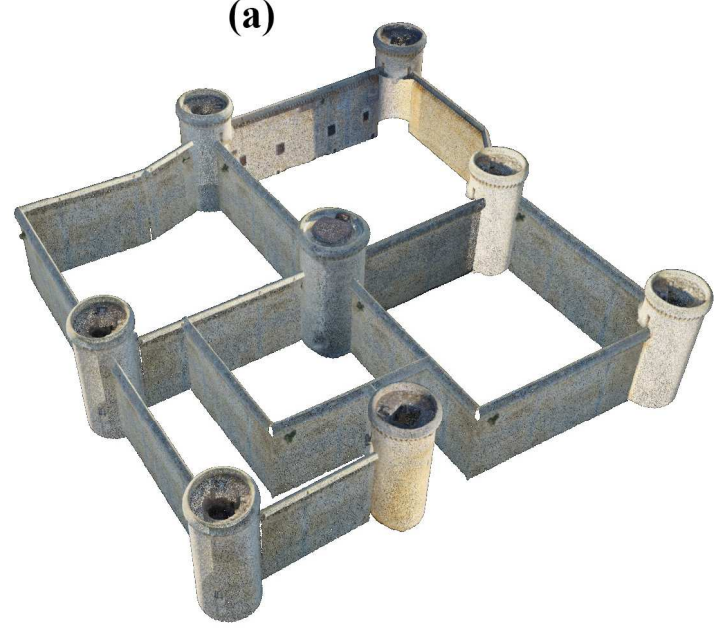

(c)

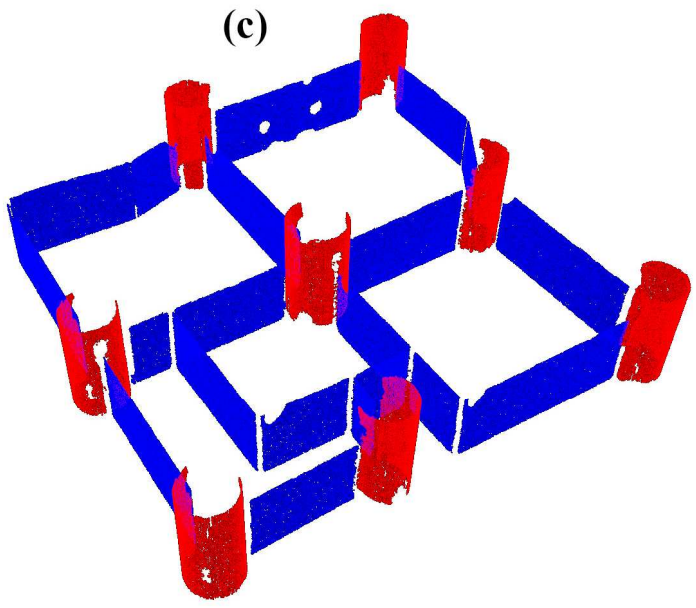

(b)

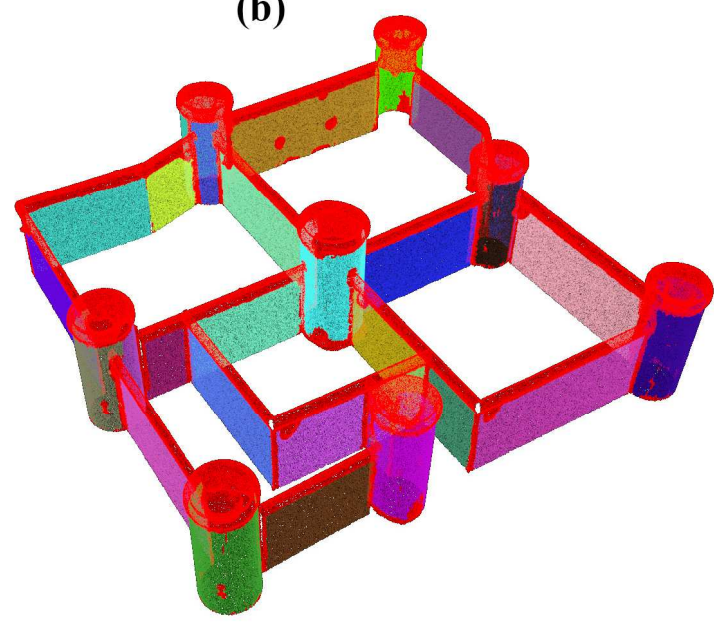

(d)

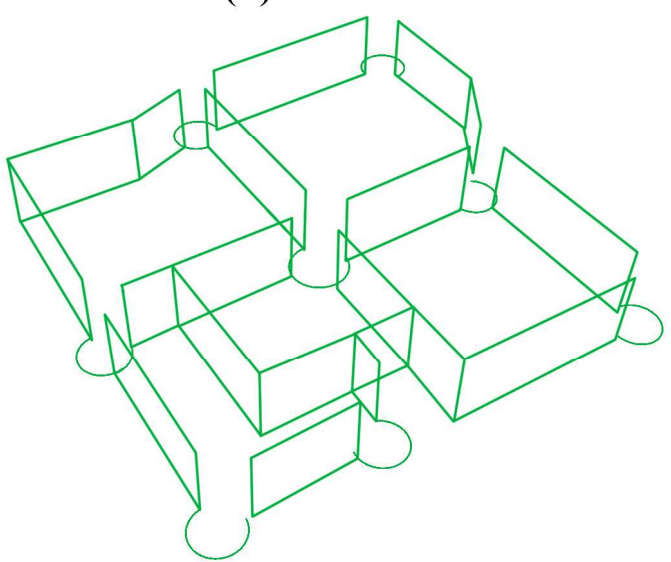

Figure 5: Synthetic point cloud. a) Pre-processed point cloud; b) segmented; c) Classified; d) Final model

As seen in Table 3, the proposed algorithm is truthfully successful in detecting the walls and towers within the point cloud and all the segments are recognized correctly in terms of its assigned category. With regard to the accuracy of the model, Lerones (2010) pointed out that a geometric resolution of $15 \mathrm{~mm}$ during the acquisition process is precise enough. Taking into account this figures, it is been assumed that a modelling error in the order of magnitude of a little few centimetres can be acceptable for an as-built 3D model. Based on this considerations, it can be stated that the geometrical accuracy of 
the model, represented by the mean distance of the points to their fitted model (see Table 3), is precise enough since the planes have an overall mean distance of $24 \pm 1 \mathrm{~mm}$ and the cylinders have a slightly larger mean distance of $64 \pm 2 \mathrm{~mm}$ which can be explained by the higher complexity of their parametrization but that still falls in the proposed range of distances.

In the other hand, the reconstructed model still has some remaining errors, which may be caused by limitations in the proposed methods. Firstly, three towers were segmented down the midline (marked with $*$ in Table 3 ) and registered as two different towers. Secondly, the intersection algorithm was not able to detect one of the intersections between a plane and the cylinders. It is noteworthy that the proposed method relays on manual parameter tuning and therefore is still human-error prone. This drawback does not affect the overall result since the parametrization step is robust enough to extract the main features of the detected primitives even with incomplete information, as seen in Figure 5d.

\section{CONCLUSIONS}

This paper presented a novel method for the parametrization of architectonic features of immovable heritage assets using point clouds as suitable input data. The proposed method was evaluated using samples of the photogrammetric 3D reconstruction of the Castle of Torrelobatón showing the feasibility of the proposed methodology. The different implemented algorithms produced satisfactory results during the analysis and parametrization stages, yielding accurate connectivity between planes (walls) and cylinders (round towers). The effective outcome of the parametrization stage, based on a projection based method, supported the chosen workflow since the final 3D model was accurate enough based on the defined metrics.

The implemented pipeline suggests many avenues for future research. The basic set of mathematical primitives, comprised of planes and cylinders, can be further increase adding cones or spheres to analyse more advanced elements such as covered towers or domes respectively in a similar projecting-based approach increasing the set of architectonic features detected. Moreover, the final model provided by the algorithm could be further enriched by implementing an opening-detection system further than latest methods that are only applicable to planar façades. Finally, the segmentation of the cylinders could be improved in order to detect a unique tower instead of several portions of the same architectonic element.

\section{ACKNOWLEDGEMENTS}

This work is partially developed under the INCEPTION project (Inclusive cultural heritage in Europe through $3 D$ semantic modelling), funded from the European Union's Horizon 2020 research and innovation programme under grant agreement No 665220 .

\section{REFERENCES}

Arayici, Y., Counsell, J., Mahdjoubi, L., Nagy, G. A., Hawas, S., Dweidar, K. (Eds.) 2017. Heritage Building Information Modelling. Routledge (UK).

Barrile, V., Bilotta, G., Meduri, G. M., 2015. Comparison between techniques for generating 3D models of cultural heritage. In proceedings International Conference on Civil Engineering, Zakynthos Island, Greece, July 16-20, 2015.

Bruno, S., De Fino, M., Fatiguso, F., 2018. Historic Building Information Modelling: performance assessment for diagnosisaided information modelling and management. Elsevier, Automation in Construction, 86, 256-276.

Cheng, D., Zhang, L., Mathiopoulos, P. T., Huang, X., 2014. A Methodology for Automated Segmentation and Reconstruction of Urban 3-D Buildings from ALS Point Clouds. IEEE, Journal of Selected Topics in Applied Earth Observations and Remote Sensing, 1(10), 4199-4217

Fischler, M. A., Bolles, R. C. 1981. Random Sample Consensus: A Paradigm for Model Fitting with Applications to Image Analysis and Automated Cartography. Communications of the ACM, 24 (6), 381-395.

Grün, A., Baltsavias, E., Henricsson, 1997. Automatic Extraction of Man- Made Objects from Aerial and Space Images, II, Birkhäuser, Basel.

Haala, N., Kada, M., 2010. An update on automatic 3D building reconstruction. ISPRS Journal of Photogrammetry and Remote Sensing, 65(6), 570-580.

Historic England, 2017. BIM for heritage: Developing a Historic Building Information Model. Swindon, Historic England. https://bit.ly/2BMlGJV. (4 February 2019)

Jung, J., Stachniss, C., Ju, S., Heo, J., 2018. Automated 3D volumetric reconstruction of multiple-room building interiors for as-built BIM. Elsevier, Advanced Engineering Informatics, $38,811-825$

Lerones, P. M., Fernández, J. L., Gil, A. M., Bermejo, J., Casanova, E. Z., 2010. A practical approach to making accurate 3D layouts of interesting cultural heritage sites through digital models. Elsevier, Journal of Cultural Heritage, 11, 1-9.

Li, Z., Zhang, L., Mathiopoulos, P. T., Liu, F., Zhang, L., Li, S., Liu, H. 2017. A hierarchical methodology for urban facade parsing from TLS point clouds. ISPRS Journal of Photogrammetry and Remote Sensing, 123, 75-93.

López, F. J., Lerones, P. M, Llamas, J., García-Bermejo, J. G., Zalama, E., 2018. Semi-automatic generation of BIM models for cultural heritage. International Journal of Heritage Architecture, 2(2), 293-302.

López, F. J., Lerones, P. M., Llamas, J., García-Bermejo, J. G., Zalama, E., 2017. A framework for using point cloud data of heritage buildings towards geometry modeling in a BIM context: a case study on Santa Maria la Real de Mave Church. International Journal of Architectural Heritage, 11(7), p. 965986.

Macher, H., Landes, T., Grussenmeyer, P., 2017. From point clouds to building information models: 3D semi-automatic reconstruction of indoors of existing buildings. MDPI, Applied Sciences, 7(10), 1030.

Ochmann, S., Vock, R., Wessel, R., Klein, R., 2015. Automatic reconstruction of parametric building models from indoor point clouds. Elsevier, Computer \& Graphics. 54, 94-103. 
Ochmann, S., Vock, R., Wessel, R., Tamke, M., Klein, R., 2016. Automatic generation of structural building descriptions from 3D point cloud scans. SCITEPRESS, In Proceedings of the 9th International Conference on Computers Graphics Theory and Applications, Lisbon, Portugal, 1, 120-127

Peternell, M., Steiner, T., 2004. Reconstruction of piecewise planar objects from point clouds. CAD Computer Aided Design, 36(4), 333-342.

Pocobelli, D. P., Boehm, J., Bryan, P., Still, J., Grau-Bové, J., 2018. BIM for heritage science: a review. Springer Open, Heritage Science.

$\mathrm{Pu}$, S., Vosselman, G., 2007. Extracting windows from terrestrial laser scanning, ISPRS Workshop on Laser Scanning 2007 and SilviLaser 2007, XXXVI-3, Espoo, Finland, September 12-14.

$\mathrm{Pu}$, S., Vosselman, G., 2009. Knowledge based reconstruction of building models from terrestrial laser scanning data, ISPRS Journal of Photogrammetry and Remote Sensing, 64 (6), 575584.

Stambler, A., Huber, D., 2014. Building modelling through enclosure reasoning. IEEE, 2014 Second International Conference on 3D Vision, Tokyo, Japan, December 8-11.

Tommasi, C., Achille, C., Fassi, F., 2016. From point cloud to BIM: A modelling challenge in the cultural heritage field. ISPRS, International Archives of the Photogrammetry, Remote Sensing and Spatial Information Sciences, XLI-B5, 429-436.

Truong-Hong, L., Laefer, D. F., Hinks, T., Carr, H., 2012. Flying Voxel Method with Delaunay Triangulation Criterion for Façade/Feature Detection for Computation. Journal of Computing in Civil Engineering, 26 (6), 691-707.

Wan, G., Sharf, A., 2012. Applications of Geometry Processing Grammar-based 3D facade segmentation and reconstruction. Computers and Graphics, 36, 216-223.

Wang, C., Cho, Y. K., Kim, C., 2015. Automatic BIM component extraction from point clouds of existing buildings for sustainability applications. Elsevier, Automation in Construction, 56, 1-13.

Xiong, X., Adan, A., Akinci, B., Huber, D., 2013. Automatic creation of semantically rich 3D building models from laser scanner data. Elsevier, Automation in Construction, 31, 325337.

Yang, X., Koehl, M., Grussenmeyer, P., 2018. Mest-to-bim: From segmented mesh elements to bim model with limited parameters. ISPRS, International Archives of the Photogrammetry, Remote Sensing and Spatial Information Sciences, XLII-2, 1213-1218.

Zolanvari, S. M., Laefer, D. F., 2016. Slicing Method for curved façade and window extraction from point clouds. ISPRS Journal of Photogrammetry and Remote Sensing, 119, 334-346.

Zolanvari, S. M., Laefer, D. F., Natanzi, A., 2018. Threedimensional building façade segmentation and opening area detection from point clouds. ISPRS International Archives of the Photogrammetry and Remote Sensing, 143, 134-149. 